\title{
EFFECT OF FIBER DISTANCE ON VARIOUS SAC-OCDMA DETECTION TECHNIQUES
}

\author{
Vandana Nath ${ }^{1}$, Nakul Jain ${ }^{2}$, Sandeep Dogra ${ }^{3}$ \\ ${ }^{1}$ Assistant Professor, Electronics and Communication Engineering, ${ }^{2,3}$ Student, M.Tech Digital and wireless \\ communication, University School of Information and Communication Technology, Guru Gobind Singh Indraprastha \\ University, Delhi, India.vandanausit@gmail.com,er.nakul29jain@gmail.com, sandeep.dogra1589@gmail.com
}

\begin{abstract}
Optical code division multiple access system (OCDMA) has been gaining importance with increasing demands of high speed and large capacity for communication in optical networks. OCDMA system is totally asynchronous, that does not require any clock signals for synchronization in the network. Therefore, OCDMA provide a network that is simpler and offers the potential for scalability to higher levels of connectivity. OCDMA encoding /decoding process also provides a level of security directly implemented in the physical layer. Using OCDMA technique high spectral efficiency is achieved, hence fiber bandwidth is used very efficiently with throughput in excess of Tbit/s. Among several kinds of OCDMA systems, spectra amplitude coding (SAC) scheme attracts increasing interest because multiple access interference (MAI) can be eliminated and preserve the orthogonality between users in the system. This paper presents comparison of three important SAC-OCDMA detection techniques, namely - Direct detection, Complementary subtraction and AND subtraction. The design of encoder and decoder modules for SAC-OCDMA system used in this paper is based on Fiber Bragg Gratings (FBGs). Here conventional single mode fiber (SMF) is used as the transmission link and the performance metric studied is Quality factor $(Q)$ in multiple access environments of various user systems for these three detection techniques. Finally Effect of increasing number of fiber distance on the direct detection technique using NRZ and RZ data formats have been studied. Simulated results show that AND subtraction technique gives better Quality-factor $(Q)$ than the complementary subtraction and direct detection techniques for the signal with same power.
\end{abstract}

Index Terms: OCDMA (optical CDMA), BER (bit error rate), MAI (multiple access interference), DW (double weight code), SAC (spectral amplitude coding) and $Q$ (quality factor)

\section{INTRODUCTION}

OCDMA system is being used for high quality video transmission due to its large bandwidth and less attenuation [1]. OCDMA system has many advantages over conventional optical systems such as more privacy to every user, flexibility in channel allocation [2]. Further it can operate asynchronously and network capacity is not limited but highly scalable [3]. The robustness of CDMA technique in wireless network is yet to prove that OCDMA offers security advantages over other multiple access systems by maintaining the quality of services [4].

OCDMA is a multiplexing technique by which each communication channel is distinguished by a specific optical code rather than a wavelength or a time slots. An encoding operation optically transforms each data bit before transmission as shown in Fig-1. Here we provide distinguishable and unique code to every user at the transmitter like: $\mathrm{C} 1, \mathrm{C} 2 \ldots, \mathrm{Cn}$ and at the receiver, the reverse decoding operation is required to recover the original data [5].

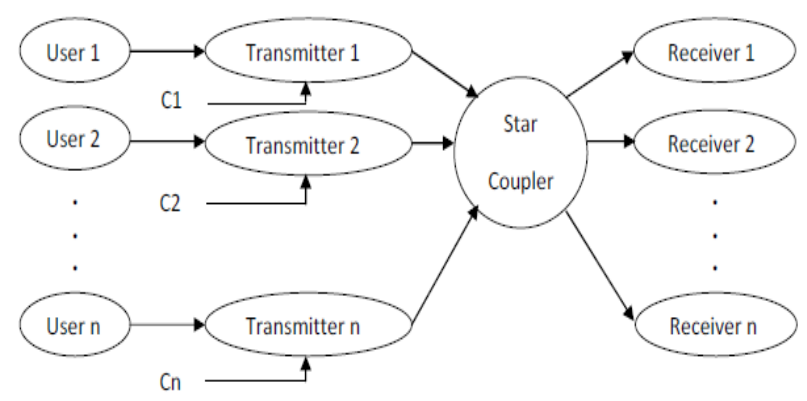

Fig-1: A General Structure of Optical code division multiple access system

SAC-OCDMA is a good candidate for optical multiple access networks over other OCDMA techniques because of its ability to reduce MAI [6]. A system consisting of unipolar sequences in the signature code is called incoherent system. System that uses bipolar codeword is called a coherent system. The incoherent source appears as a good candidate for SAC as it is inherently broadband, a necessary characteristic of SAC [7]. 


\section{VARIOUS DETECTION TECHNIQUES}

\subsection{Direct detection technique}

Direct implementation of DW code is shown in Fig-2. In this technique only those component of the optical spectrum are retained which are desired. Other undesirable components are removed by filtering. In direct detection technique MAI does not exists. But, this technique is applicable only to those codes, in which the spectral chips are not overlapped with other spectral chips of the other channel, i.e. a minimum of one clean chip in every code sequence. From Fig-2 it is clear that same frequency component being directly detected at the receiver side and hence its circuitry is very simple and less costly.

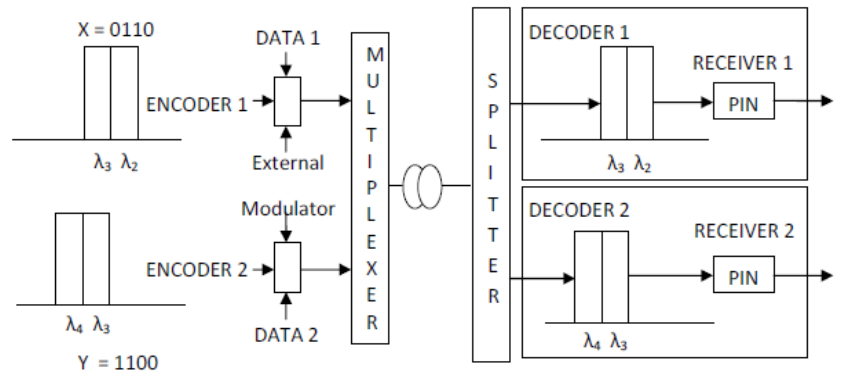

Fig-2: Implementation of the Direct Detection Technique

\subsection{Complementary Subtraction Technique}

Complementary detection technique is also known as balance detection technique [8]. In complementary subtraction technique, the cross-correlation is defined as:-

$\theta_{X Y}(k)=\sum_{i=0}^{N-1} x_{i} y_{i+k}$

Where $\mathrm{X}$ and $\mathrm{Y}$ are two OCDMA code sequences, the complement of sequence $(\mathrm{X})$ is given by $(\bar{X})$ whose Elements are obtained from $(\mathrm{X})$ by $\bar{X}=1-\mathrm{X}$.

Let $\mathrm{X}=0011$ and $\mathrm{Y}=0110$ and therefore $\bar{X}=1100$. The periodic cross correlation sequence between $(\bar{X})$ and $(\mathrm{Y})$ is similar to Equation (1) and is expressed as:

$\theta_{\bar{X} Y}(k)=\sum_{i=0}^{N-1} \bar{x}_{i} y_{i+k}$

We want the sequence to be

$\theta_{X Y}(k)=\theta_{\bar{X} Y}(k)$

Now at the receiver, the photo detectors will detect the two complementary inputs which will be fed to the subtractor whose cross-correlation output, $\mathrm{Z}$ can be expressed as:

$Z_{\text {Complementary }}=\theta_{X Y}(k)-\theta_{\bar{X} Y}(k)$

After solving equation (4) if we get 0 , at the output of the subtractor, there will be no more cross-correlation terms indicating that there is no more signal from other users in the intended channel and hence there will be no more MAI.

Transmitter of Complementary model is similar to that of direct detection. In receiver, the received signal is divided into two complementary branches of spectral chips as shown in Fig-3. These two branches of spectral signals are sent to a balanced detector that computes the correlation difference.

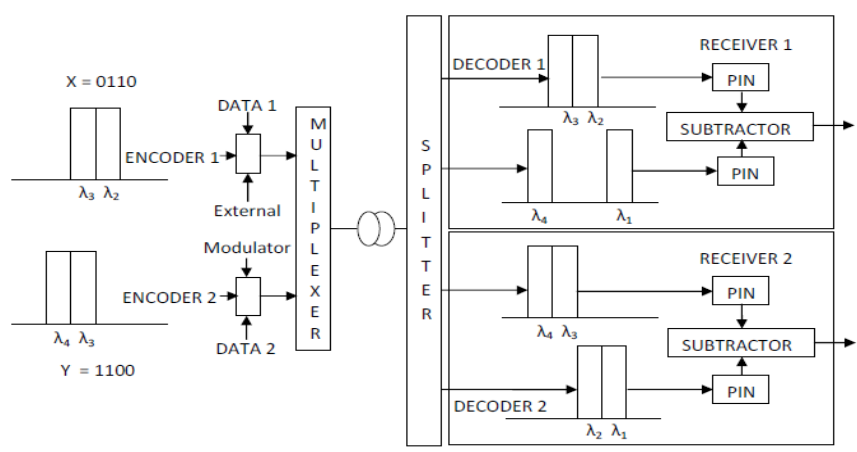

Fig-3: Implementation of the Complementary Subtraction Technique [9]

\subsection{AND Subtraction Technique}

AND subtraction technique, have the cross-correlation $\theta_{\bar{X} Y}(k)$ is substituted by $\theta_{(X \& Y) Y}(k)$ where the $\theta_{(X \& Y)}$ represents the AND operation between sequences $\mathrm{X}$ and $\mathrm{Y}$.

Here at the receiver:

$Z_{A N D}=\theta_{X Y}(k)-\theta_{(X \& Y) Y}(k)$

Equation (5) shows that, with AND subtraction technique, the MAI or the interference from other channels can also be cancelled out.

In this technique, the received signal is divided into two AND branches of spectral chips as shown in Fig-4.

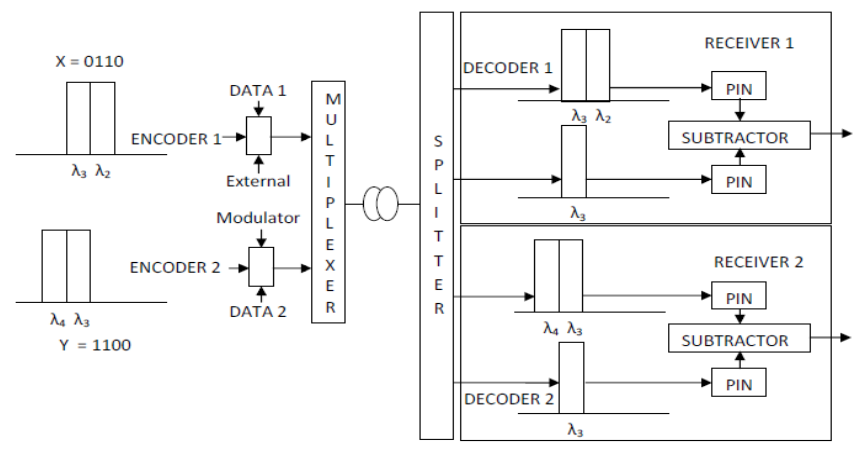

Fig-4: Implementation of the Complementary Subtraction Technique [9] 


\section{EXPERIMENTAL SETUP}

Direct detection using NRZ and RZ data formats have been simulated using Optisystem 11 as shown in Fig-5.

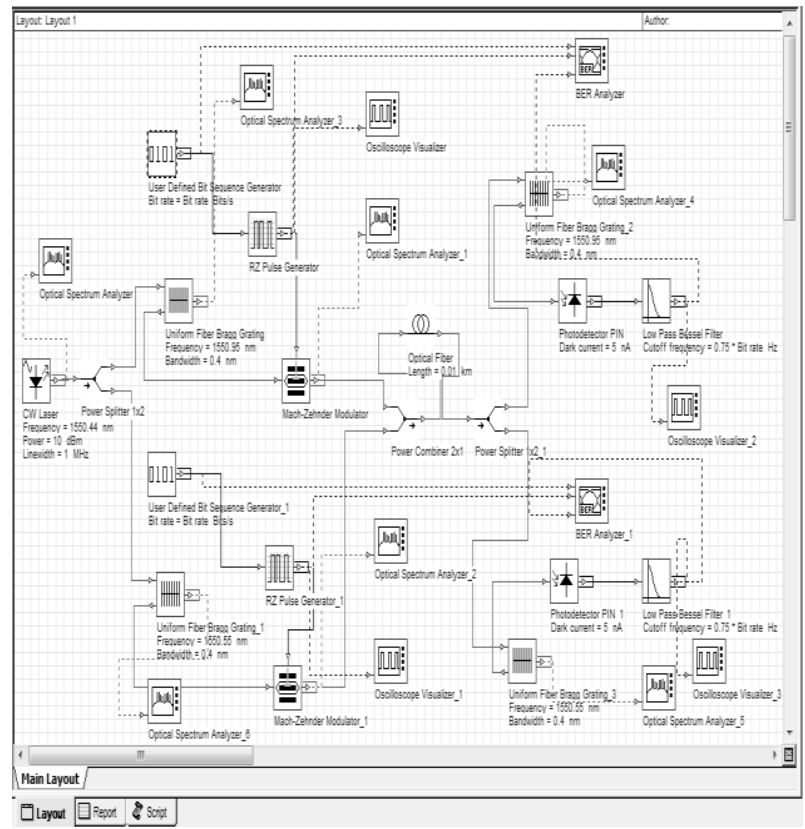

Fig-5: Layout design of OCDMA system using Direct Detection with RZ data format

And subtraction and complementary subtraction techniques have also been simulated in the similar manner. Various parameters used for this simulation for three users using Walsh Hadamard code are given in Table-1.

Table-1: Simulation parameters used for the simulation of the OCDMA system

\begin{tabular}{|c|c|}
\hline Parameters & Value \\
\hline \multicolumn{2}{|c|}{ White light specification } \\
\hline White light source wavelength & $1550.5 \mathrm{~nm}$ \\
\hline White light source power & $-115 \mathrm{dBm}$ \\
\hline \multicolumn{2}{|c|}{ Optical fiber } \\
\hline Type of optical fiber & $\begin{array}{c}\text { The ITU-T G.652 } \\
\text { standard single } \\
\text { mode optical fiber. }\end{array}$ \\
\hline \multicolumn{2}{|c|}{ Fiber brag grating specifications } \\
\hline FBG cut off wavelength $\lambda_{0}$ & $1548.5 \mathrm{~nm}$ \\
\hline FBG cut off wavelength $\lambda_{1}$ & $1550.1 \mathrm{~nm}$ \\
\hline FBG cut off wavelength $\lambda_{2}$ & $1550.9 \mathrm{~nm}$ \\
\hline FBG cut off wavelength $\lambda_{3}$ & $1552.5 \mathrm{~nm}$ \\
\hline Other parameters \\
\hline Bandwidth & $.6 \mathrm{~nm}$ \\
\hline Reflectivity & .9998 \\
\hline
\end{tabular}

\section{RESULTS AND DISCUSSIONS}

The performance evaluation of the OCDMA system using Direct Detection method with two types of data formats i.e. NRZ and RZ, has been checked on the basis of BER and fiber length as shown in Fig-6. For this purpose fiber length is varied from $10 \mathrm{~m}$ to $10 \mathrm{Km}$, other parameters like source power and bit rate are kept constant.

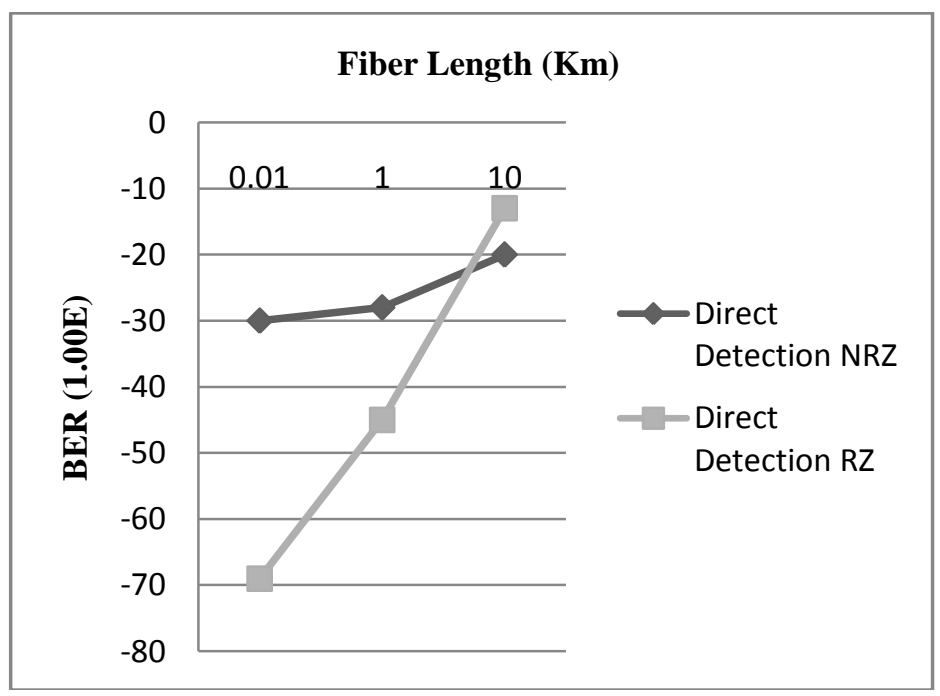

Fig-6: BER Vs Fiber Length Using NRZ and RZ Direct Detection Techniques

Fig-6 shows that an increase in fiber length causes increment in dispersion of the input signal, so BER also increases with distance, also it is clear from the above graph that for small distance, RZ data format is better as compared to NRZ data and vice versa for larger distance. Here BER is taken as $1.00 \mathrm{E}$ and data on $\mathrm{Y}$-axis shows the factor $\mathrm{E}$ power.

To differentiate between the three detection techniques simulation is carried out with $10 \mathrm{~km}$ optical fiber and data rate 200 mbps. Fig.7 presents comparison of Q-factor for three detection techniques. It clearly shows that AND detection technique has maximum Q-factor. 


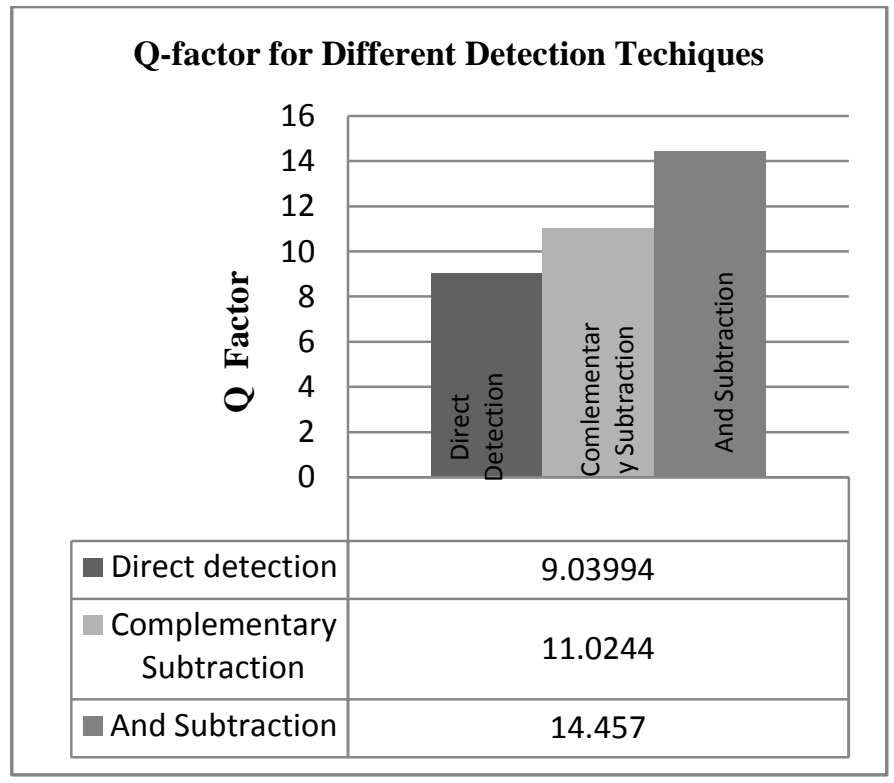

Fig-7: Q-factor of detection technique for one user for $10 \mathrm{~km}$ optical fiber

\section{CONCLUSIONS}

Direct detection, Complementary subtraction and And Subtraction SAC- OCDMA have been studied and implemented. It has been observed that performance of OCDMA system decreases as the distance of fiber increases in all the three techniques i.e. lesser the distance better is the transmission. Direct detection technique with RZ data format for smaller distance and with NRZ data format for longer distance yields better results and is the simplest and most economical techniques as the number of components used are very less as compared to other two techniques. Q-factor has been observed for different detection techniques which shows that AND subtraction detection technique gives the better performance than direct detection and complementary subtraction techniques.

\section{REFERENCES:}

[1] F. A. Hatim, F. N. Hasoon, S. A. Aljunid, and S. Shaari, "Optimum Transmit Power for Optical CDMA Transmission Systems Considering Nonlinear Stimulated Brillouin Scattering (SBS) Effects", International Conference on Electronic Design, Penang, Malaysia, 2008.

[2] P. Li and W. Hamouda, "Performance of multiple-input and multiple output orthogonal frequency and code division multiplexing systems in fading channels", The

Institution of Engineering and Technology, vol. 5, no.1, pp. 1$11,2011$.

[3] A. A. Garba and J. Bajcsy, "Optical Code Division Multiple Access Network Transmission With M-ary Chip Symbols", Optical Communication Network, vol. 3, no. 5, pp. 435-446, 2011.

[4] R J. Salehi, "Code Division Multiple Access Techniques in Optical Fiber Networks-Part 1: Fundamental Principles", IEEE Transactions on Communications, vol. 37, pp. 824-833, 1989.

[5] M. S. Ab-Rahman and M.F.A. Shaarani, "The proposal of OCDMA encoder based on optical cross and add-and-drop multiplexer (OXADM)-device characteristic", International Journal of Computer Science and Network Security, vol.8, no.12, pp.259-266, December 2008.

[6] Z. Wei, H. M. H Shalaby, and H. Ghafauri-Shiraz, "Modified quadratic congruence codes for fiber Fiber-BraggGrating-based spectral amplitude- coding optical CDMA systems," Journal of Lightwave Technology, vol. 19, pp. 12741281, 2001.

[7] H. A. Fadhil, S. A. Aljunid, and R. B. Ahmad. " Design considerations of high performance optical code division multiple access: a new spectral amplitude code based on laser and light emitting diode light source", The institution of engineering and technology, vol. 4, no. 1, pp. 29-34, May 2010. [8] R. Hui, et al., Subcarrier Multiplexing for High Speed Optical Transmission, Journal of Lightwave

Technology, Vol. 20, no. 3, pp. 417-427, 2002.

[9] C.M. Negi, Amit Pandey, Gireesh G. Soni, Saral K. Gupta and J. Kumar, " Optical CDMA Networks Using Different Detection Techniques and Coding Schemes", International Journal of Future Generation Communication and Networking Vol. 4, No. 3, September, 2011.

\section{BIOGRAPHIES:}

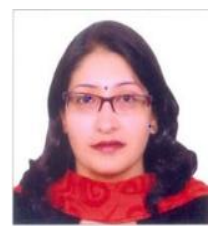

Vandana Nath is Assistant Professor in University school of Information and Communication technology, Guru Gobind Singh Indraprastha University Delhi, India. She received her doctorate from University of Delhi, India. She received her Master's from GGSIPU, Delhi. She has published 15 papers in various International journals and conferences. She has around 12 years of Research and teaching experience. Her areas of specialization are Analog Electronics, Optical communications, Information Theory \& coding.

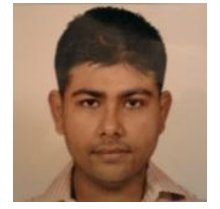

Nakul Jain is student of M.Tech in Digital and wireless communication from USICT, GGSIPU, Delhi, India. He has done his B.Tech (Electronics and communication) from Bhagwan Parshuram Institute of Technology. His areas of interest include Optical Communication, Mobile Communication and Embedded Systems.

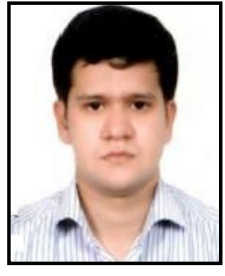

communication
Sandeep Dogra is student of M.Tech in Digital and wireless communication from USICT, GGSIPU, Delhi, India. He has done his B.Tech (Electronics and communication) from Bhagwan Parshuram Institute of Technology. His areas of interest include optical communication and spread spectrum 